\title{
Piloting Electronic Case Reporting for Improved Surveillance of Sexually Transmitted Diseases in Utah
}

\author{
Amanda Whipple1, Joseph Jackson ${ }^{1, *}$, Joshua Ridderhoff ${ }^{2}$, Allyn K. Nakashima1 \\ 1. Utah Department of Health, Salt Lake City, UT, USA \\ 2. Utah Department of Technology Services, Salt Lake City, UT, USA
}

\begin{abstract}
Objectives: The Utah Department of Health (UDOH) developed an electronic case reporting (eCR) process to automatically transfer clinical data from a provider to the state health department, with aims of improving sexually transmitted disease (STD) surveillance data quality, decreasing the time spent on STD case investigations, and expanding the process to other diseases and larger healthcare systems.
\end{abstract}

Methods: Reportable Conditions Trigger Codes (RCTC) were placed into the electronic health record (EHR) system at Planned Parenthood Association of Utah (PPAU) to trigger the automatic transfer of clinical data to Utah's public health surveillance system. Received data were de-duplicated, processed, and assigned directly to the public health surveillance system, with minimal manual intervention.

Results: Eighteen new data elements, important for STD case investigations, were transferred to cases with eCR. Additionally, the clinical time spent transmitting data was vastly reduced. With the new eCR process more complete and timely data is received by public health. Providers, as well as public health, now spend less time manually transmitting clinical data by fax and/or phone.

Discussion: Automated processes are challenging but can be achieved with a robust disease surveillance system, flexible rules engine, skillful programming, on-going analysis, and successful partnerships. The eCR process created for this project can potentially be useful for other conditions outside of STDs.

Conclusion: Results of this demonstration project offer an opportunity for readers to learn about eCR and apply lessons learned to improve their existing eCR systems, or future public health informatics initiatives, at any state-level jurisdiction.

Key Words: Electronic Case Reporting, eCR, Electronic Health Record, EHR, STD Surveillance

Abbreviations: Application Programming Interface (API), Chlamydia Trachomatis (CT), Clinical Document Architecture (CDA), Consolidated Clinical Document Architecture (C-CDA), Continuity of Care Document (CCD), Electronic Case Reporting (eCR), Electronic Health Record (EHR), Electronic Laboratory Reporting (ELR), Electronic Message Staging Area (EMSA), Extensible Markup Language (XML), Human Immunodeficiency Virus (HIV), International Classification of Diseases (ICD), Logical Observation Identifiers Names and Codes (LOINC), Neisseria Gonorrhoeoa (GC), Planned Parenthood 
Association of Utah (PPAU), Reportable Conditions Trigger Codes (RCTC), Secure File Transfer Protocol (SFTP), Sexually Transmitted Disease (STD), Systemized Nomenclature of Medicine (SNOMED), STD Surveillance Network (SSuN), Time-Motion (T-M), Utah Department of Health (UDOH).

*Correspondence: joejackson@utah.gov

DOI: 10.5210/ojphi.v11i2.9733

Copyright @2019 the author(s)

This is an Open Access article. Authors own copyright of their articles appearing in the Online Journal of Public Health Informatics. Readers may copy articles without permission of the copyright owner(s), as long as the author and OJPHI are acknowledged in the copy and the copy is used for educational, not-for-profit purposes.

\section{Introduction}

Case reports of reportable sexually transmitted diseases (STDs) and additional clinical information needed to conduct and close case investigations have historically been manually recorded on paper and sent to the Utah Department of Health (UDOH) through fax or email. This process can yield incomplete or inaccurate reports and often requires extensive and time-consuming follow-up by local health department (LHD) investigators to obtain all the information necessary to sufficiently complete case investigations [1,2].

With the introduction of electronic laboratory reporting (ELR) in Utah in 2013, laboratory reports of disease were available to UDOH more quickly than with a paper-based and manual data entry system. ELR is now implemented in many laboratories and state health departments nationwide, and although the ELR process greatly reduces the burden of disease reporting for laboratories and providers, information needed for public health investigations to complete case reports is often still incomplete or missing altogether; therefore, follow-up with providers, as well as patients, may be necessary. In order to adequately close STD cases in Utah, further investigation must often be performed to collect complete information on patient demographics, facilities, providers, treatments, pregnancy status, and other relevant variables.

Utah is a low-morbidity state for Chlamydia trachomatis (CT), Neisseria gonorhoeae (GC), human immunodeficiency virus (HIV), and syphilis [3]; therefore, at the time of this pilot project, case investigations were initiated for all laboratory confirmed cases. The information required by UDOH to adequately close a case investigation includes data not transmitted from the main laboratory that processes the majority of Planned Parenthood Association of Utah's (PPAU's) specimens in Utah. Thus, recording the necessary case information required phone calls and faxes between Utah providers and LHD investigators for obtaining clinical data.

Many clinical providers, including PPAU, now employ electronic health record (EHR) systems in their practice. EHR data contain a wealth of patient information, including the data needed to close STD case investigations in Utah. As EHRs become more commonly used, electronic case reporting 
(eCR) as demonstrated in this report could provide an opportunity to obtain important clinical data electronically with the same automation benefits seen through ELR $[4,5]$.

\section{Background of eCR}

$\mathrm{eCR}$ is the automated process of generating and transmitting case report data from EHRs to public health. eCR affords the opportunity to enhance data obtained through ELR while eliminating the need to manually acquire clinical data for epidemiologic investigations. eCR shows promise for capturing more complete and accurate clinical data as well as timelier detection and reporting of cases [6]. Additionally, eCR can facilitate the formation of a robust and adaptable infrastructure to support rapid reporting of emerging conditions [2].

One common way eCR data are transmitted is through a Continuity of Care Document (CCD). $\mathrm{CCD}$ is one of the document types defined by consolidated clinical data architecture (C-CDA), a standardized method of implementing the clinical data architecture (CDA) standard [7]. By using standard Extensible Markup Language (XML) data elements defined by CDA, a CCD records a patient's history of care including procedures performed, clinical diagnoses, and prescribed medications in a standardized format. Reporting facilities using a Meaningful Use Stage 2compliant EHR system can automatically transmit these data to public health via a CCD where they can be consumed by, and imported into, a systematized electronic messaging interface to extract clinical data important for case reports, case investigations, and case closures.

\section{Goals of eCR Implementation in Utah}

Through a competitive process, Utah secured funding through the STD Surveillance Network (SSuN) supported by the Centers for Disease Control and Prevention (CDC) to demonstrate the feasibility of implementing an eCR process with PPAU, a high-volume reporter of CT and GC cases in Utah. The goals of the SSuN demonstration project were to route EHR data from PPAU directly to UDOH's public health surveillance system (EpiTrax $\left.{ }^{\circledR}\right)$, with aims of improving STD surveillance data timeliness and completeness, decreasing the time spent on STD case investigations, and expanding the eCR infrastructure to other diseases and larger healthcare systems in the future.

\section{Methods}

As the CCD is a natively exportable document from Meaningful Use Stage 2-compliant EHR systems, the most functional way to obtain the necessary clinical data was to have PPAU's EHR vendor configure a daily export of CCDs based on implemented reportable conditions trigger codes (RCTCs). Once an RCTC was detected, the EHR system at PPAU (NextGen ${ }^{\circledR}$ ) was triggered to automatically send the relevant CCD to UDOH through a secure file transfer protocol (SFTP). Once received at UDOH, the CCDs were parsed into individual patient encounters, and then parsed again within the public health surveillance system by International Classification of Diseases (ICD-10), Logical Observation Identifiers Names and Codes (LOINC), and Systemized Nomenclature of Medicine (SNOMED) codes, which are diagnostic, laboratory testing, and clinical codes applied to all patient encounters. Any records found with ICD-10, LOINC, and/or 
SNOMED codes specific to CT or GC were then processed through the Electronic Message Staging Area (EMSA). EMSA is a sophisticated rules engine that de-duplicates cases, determines diseases and conditions, completes person-matching functions, and links laboratory results to disease cases. A detailed explanation of how the actual transport process was achieved is described below.

\section{Message Processing}

LOINC, ICD-10, and SNOMED triggers based on the RCTC were implemented in PPAU's EHR. When a patient's encounter included an identified trigger, a CCD for that patient was sent to UDOH's SFTP server. PPAU sent daily batches of all cases that contained the implemented triggers specific to CT and GC cases (HIV and syphilis cases were implemented after CT and GC, since historically PPAU has a lower volume of those diseases).

Once received, a health data interchange application (NextGen Connect integration engine) was used for initial message filtering, pre-processing, parsing, and routing. CCD documents, by design, contain the patient's entire clinical history within the reporting facility. This historical clinical data may be re-sent repeatedly over an extended period of time; thus it was vital to limit which data were routed for importation from each received CCD to reduce duplication of data in EpiTrax.

Individual patient encounters were first identified in the CCD and split into an intermediate custom XML document, known as a "Simplified CCDA Encounter," with all relevant data from the original CCD linked to that encounter included. Any encounters that were deemed to be irrelevant due to the age of the encounter in relation to the encounter of interest were filtered out. NextGen Connect was also used to translate ICD codes representing clinical diagnoses within each encounter during this processing. ICD codes indicating pregnancy were used to populate pregnancy status in the "Simplified CCDA Encounter" document. ICD codes indicating a condition of interest (CT, GC, HIV, or syphilis) were mapped to a disease area of the "Simplified CCDA Encounter." Any remaining ICD codes that did not indicate a relevant concept were discarded. The mapping of ICD codes was configured in EMSA, and any unidentified ICD codes were flagged in the EMSA interface for resolution by UDOH staff (Figure 1).

Next, each "Simplified CCDA Encounter" was routed to EMSA. EMSA is a complex web application that allows informaticists and epidemiologists to define criteria based on case definition rules to determine how electronic health messages should be used to affect case records in EpiTrax (Figure 2). EMSA uses these rules in coordination with an Application Programming Interface (API) provided by EpiTrax to perform automated person matching and determine when to create or update cases in EpiTrax for investigation or surveillance. Using these rules, EMSA organizes data associated with each condition identified via triggered LOINC, SNOMED, or ICD codes. Once EMSA has determined that it has data relevant to a condition of interest, it first attempts to locate a matching case in EpiTrax. If ambiguous search results are encountered, the message can be diverted for review by UDOH staff. If an existing case is identified, the new clinical data is compared against existing values known, and an evaluation is made as to whether the new data should update existing values or if duplicate information can be discarded. If no existing case is identified, EMSA can trigger the creation of a new case in EpiTrax. 
Finally, EMSA uses the EpiTrax API to append clinical data elements of interest to cases in EpiTrax, either via applicable defined data fields, or as case notes where necessary (Figure 3). The EpiTrax API further works to prevent duplication of data in cardinal data elements, such as phone number, address, and treatment entries. Treatment data not related to the case were also filtered out through the API.

EMSA has the ability to complete the above processes automatically and continually for nearly all of the large volume of data received by eCR. In addition to determining conditions and linking laboratory information to cases, one of the most important functions of EMSA was the ability to de-duplicate cases. On average, UDOH received 70 CCD messages daily, which were then parsed into a daily average of 235 individual sets of clinical data associated with a triggered condition. This highlights the need for a robust, automated rules engine to eliminate the need for manual review of all messages.

Figure 1: ICD Code Management Interface

Home 》 Admin 》 Vocabulary 》 Master Vocabulary 》 Dictionary Condition LOINC SNOMED PFGE ICD Rules by Condition 4 All assigned roles \ EMSA2

国国 ICD Code Manager

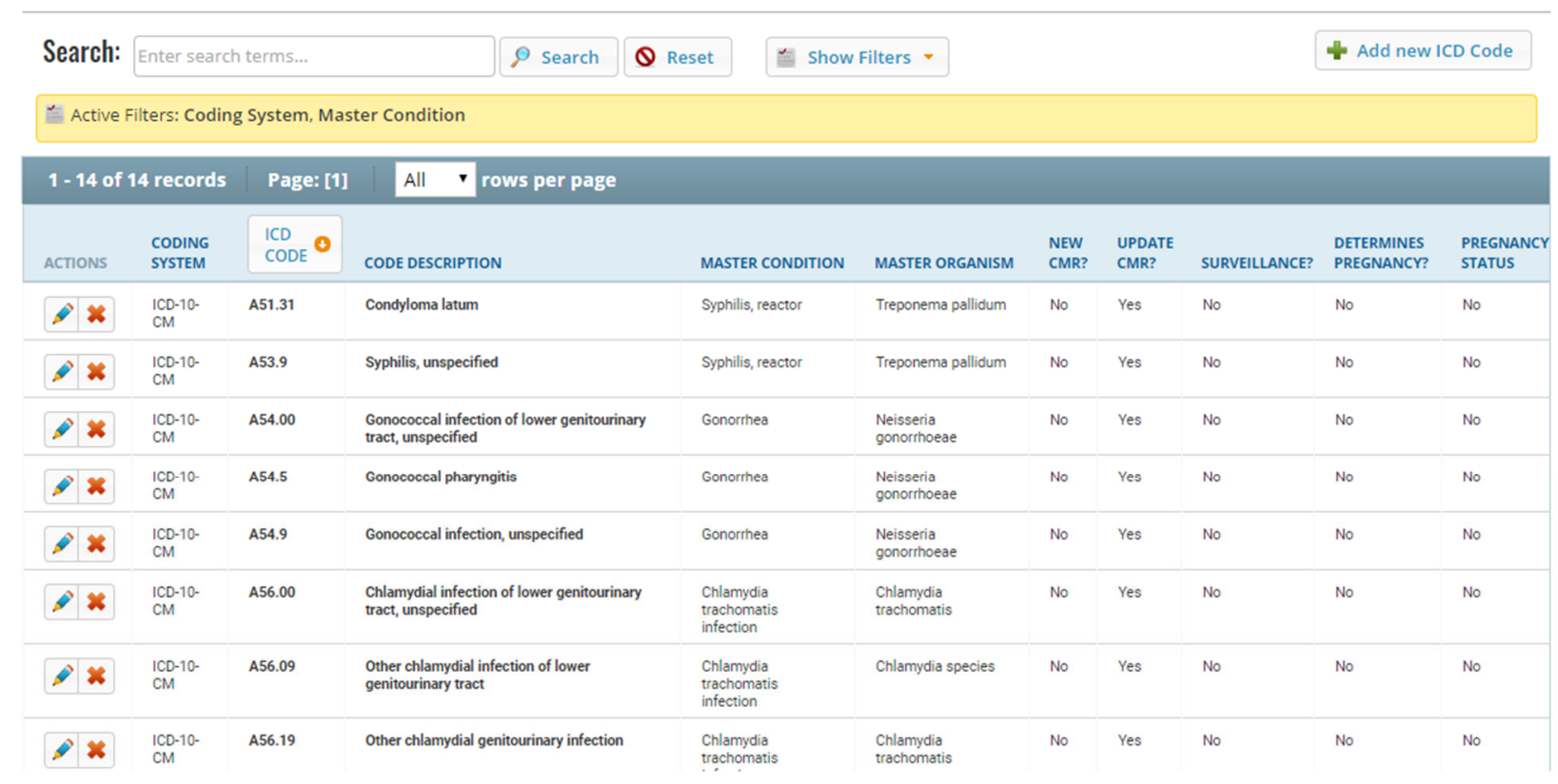


Figure 2: Condition Rules Management Interface

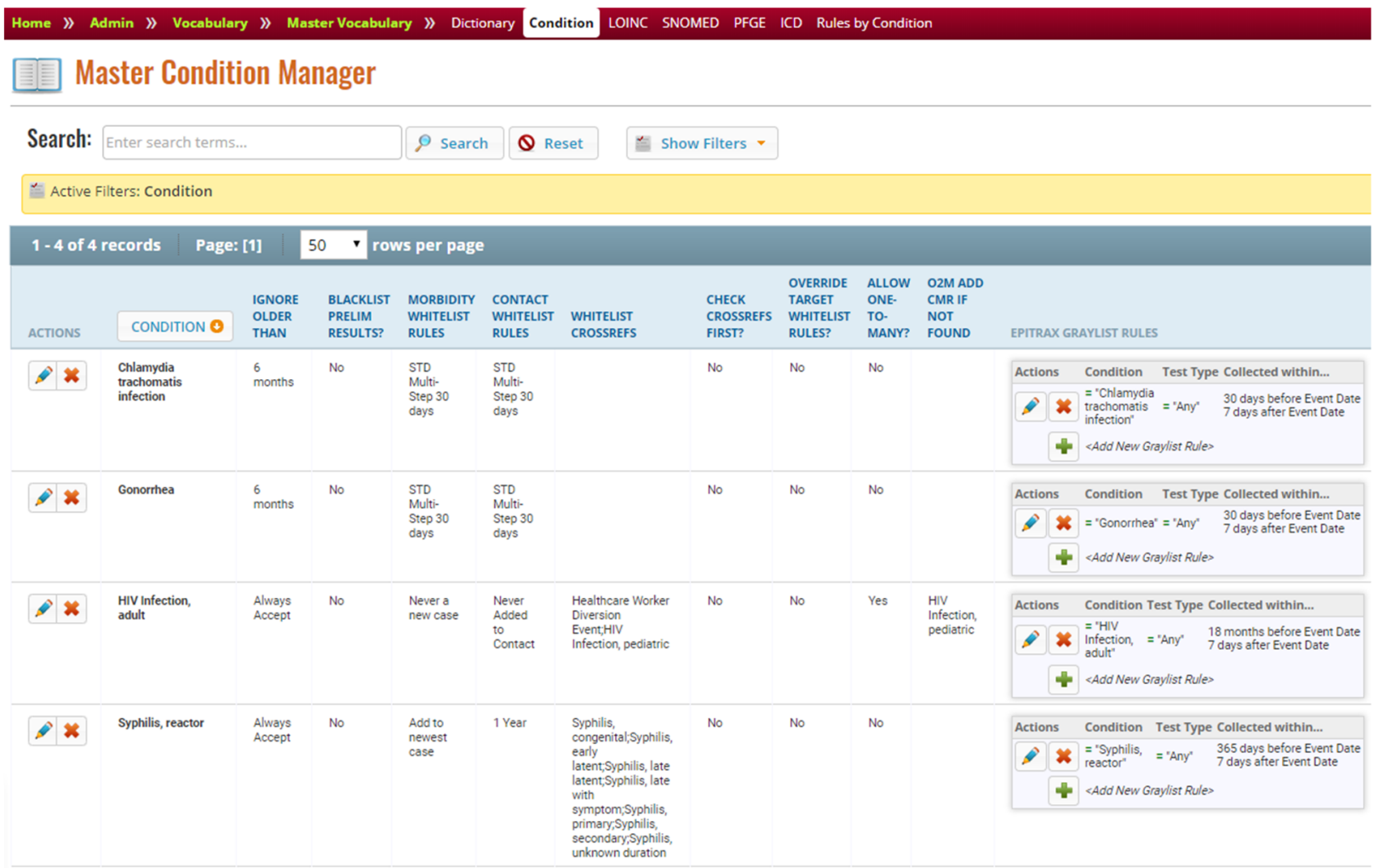


Figure 3: UDOH Electronic Message Processing Workflow

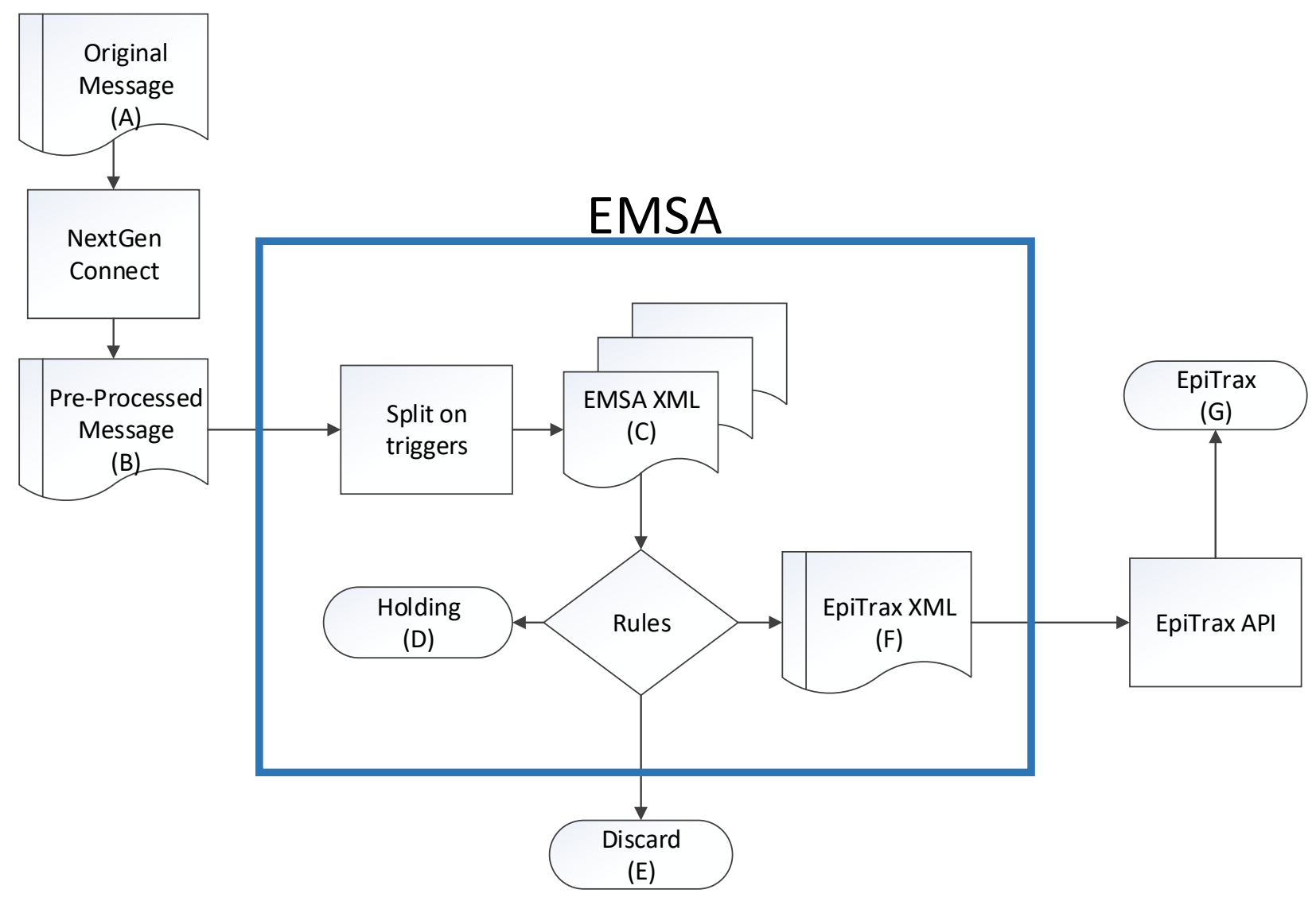

\section{Results}

Starting in October 2016, clinical data were automatically electronically transferred from PPAU's EHR to UDOH's public health surveillance system. Below are descriptions of the impact of the successful implementation of eCR on the quality of STD case reports at UDOH, including impacts on completeness, timeliness, and volume of reporting, as well as a description of the results of Time-Motion studies.

\section{Completeness}

Eighteen data elements important for STD case investigations were provided by eCR and imported into EpiTrax when information was new or updated; none of this data was previously transmitted through ELR from the laboratory that processes the majority of PPAU's STD specimens (Table 1). Among the most important elements transmitted for local health investigators were patient demographics, treatment information, pregnancy status, and facility/clinician data. 
Table 1: New eCR Data Elements

\begin{tabular}{|l|l|}
\hline Patient Street & Pregnancy Status \\
\hline Patient Unit Number & Treatment Name \\
\hline Patient City & Treatment Dosage \\
\hline Patient Zip Code & Treatment Quantity \\
\hline Patient Area Code and Phone Number & Treatment Start Date \\
\hline Patient Ethnicity & Treatment Stop Date \\
\hline Patient Race & Clinician Name \\
\hline Patient Birth Sex & Clinician Area Code and Phone Number \\
\hline Medical Record Number & Clinical Diagnosis/Condition from ICD Code \\
\hline
\end{tabular}

\section{Timeliness}

More timely information was also available through eCR. When relying solely on ELR, the average time for UDOH to obtain all the relevant clinical information necessary to close out a STD case could take over a week; however, after implementing eCR, the average time for UDOH to receive all relevant clinical information was 4 hours and 34 minutes, followed by an average time to transfer those clinical data elements into EpiTrax of 3 hours and 24 minutes. All messages were received from PPAU at UDOH within 18 hours of generation (Figure 4), with the majority of messages processed within one hour of receipt (Figure 5). Message receipt was calculated from the time the message was generated at PPAU to receipt by the UDOH integration engine. Messages taking longer than five hours to receive were batches of files delayed in transfer by system issues between PPAU and UDOH's SFTP server or the transfer from the SFTP server to the integration engine. Manual intervention was required for some messages due to receiving novel coded vocabulary terms (requiring UDOH staff to define the terminology), unexpected changes to message structure by vendors, equivocal person matching results, or incomplete data necessary to complete automated rule evaluation (in some cases requiring follow-up with providers). Messages requiring manual intervention for any of the above issues took longer to resolve and complete processing than those processed automatically; however, only 194 system messages took more than 12 hours to process. 
Figure 4: Time for UDOH to Receive CCDs from PPAU

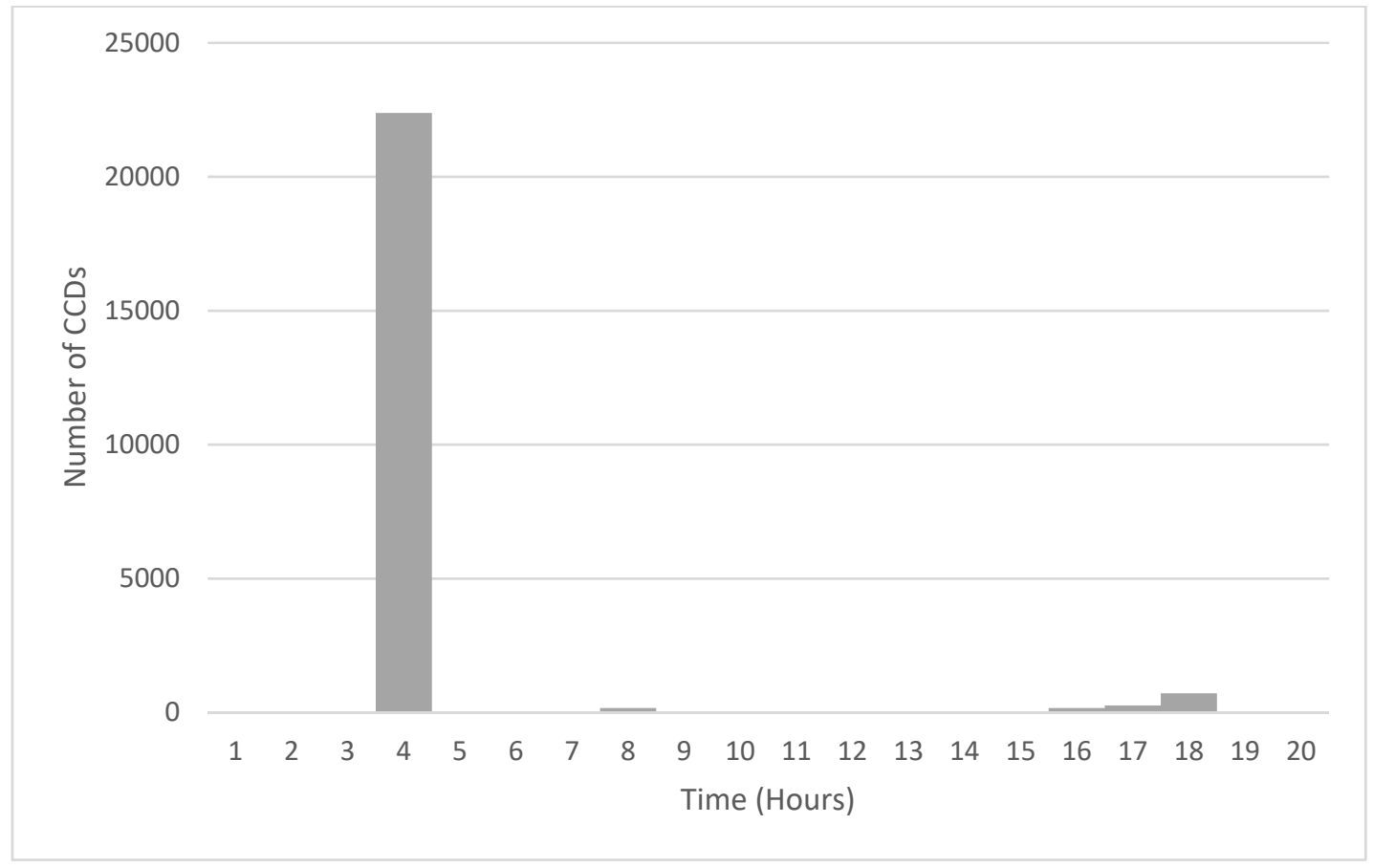

Figure 5: Time from Receipt at UDOH until Case is Updated

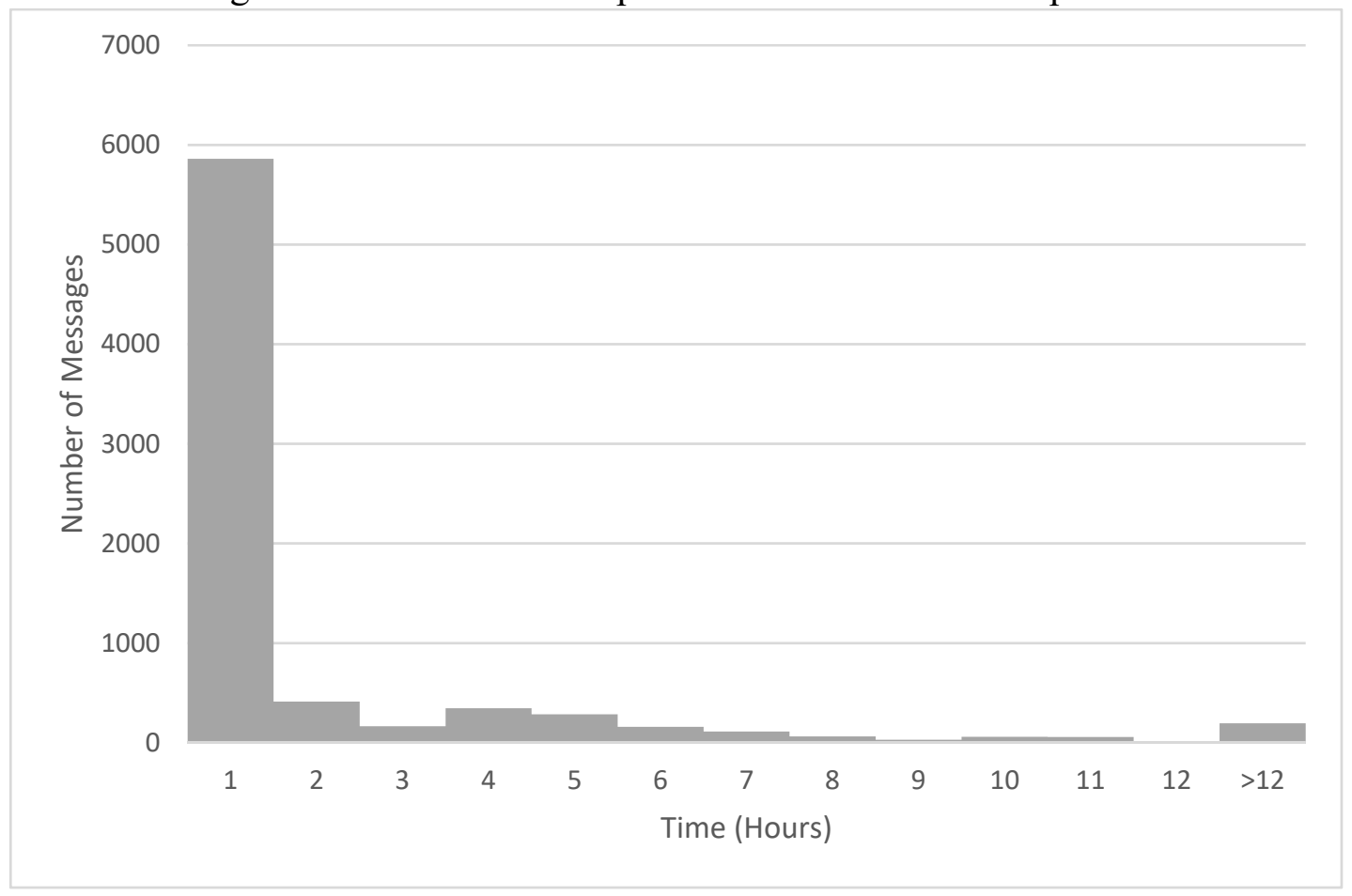




\section{Time-Motion Studies}

For this project, time-motion (T-M) studies were conducted before and after the implementation of eCR to assess time saved by automating data transfer. PPAU clinical time spent on faxes, emails, phone calls, paperwork, and EHR usage necessary to send clinical data to local health investigators was collected in 2014 prior to eCR implementation. Results of the initial T-M study showed that prior to eCR implementation, PPAU staff were conservatively spending 4.22 minutes per case of $\mathrm{CT}$ and/or GC to manually send clinical data to UDOH (HIV and syphilis were not included in the study). As PPAU diagnoses approximately 2,300 CT and GC cases yearly, this equates to nearly 162 hours being spent annually on this manual process. Results of the follow-up T-M study conducted in 2018, after the implementation of eCR, showed that the time spent by PPAU staff to transmit data to UDOH per case of CT and/or GC decreased to 0.04 minutes (2.4 seconds) per case. This equates to roughly 1.5 hours annually to transmit STD clinical data to UDOH, a time savings of approximately 161 hours per year across the eight PPAU clinics.

\section{Volume}

As of August 31, 2018, the total number of STD cases that have been updated with clinical information since the process became functional is 2,735 and increases daily. The volume for the below brief analysis is only for eight clinics (all PPAU clinics in Utah) and only four conditions (CT, GC, syphilis, and HIV). It should be noted that Utah has a non-positive laboratory reporting rule for CT, GC, syphilis, and HIV confirmatory tests, meaning that positive as well as negative, indeterminate, and inconclusive laboratory reports of the aforementioned conditions are reported to UDOH. This helps account for the large volume of CCD encounters that are moved to a holding area where the information is held for 18 months for future case matching. After 18 months the information is stripped of identifiable data but aggregate non-positive data is retained. The brief analysis of cases updated automatically with clinical data from October 1, 2017 to August 31, 2018 is as follows (Table 2):

Table 2: CCD Disposition Breakdown, 10/01/2017 - 08/31/2018

\begin{tabular}{|l|l|}
\hline CCDs received by UDOH (Figure 3 $(A))$ & 23,508 \\
\hline $\begin{array}{l}\text { CCDs containing at least one useable trigger code (ICD, LOINC, or SNOMED) } \\
\text { (Figure 3 (B)) }\end{array}$ & 19,696 \\
\hline $\begin{array}{l}\text { CCDs processed and discarded due to unusable trigger codes (i.e. relevant but generic } \\
\text { trigger codes that could not be mapped to a condition in EpiTrax) (Figure 3 (E)) }\end{array}$ & 3,812 \\
\hline $\begin{array}{l}\text { System messages created from useable trigger codes; messages were split into patient } \\
\text { encounters and split again by ICD/LOINC/SNOMED trigger codes as there could be } \\
\text { multiple trigger codes in one encounter (Figure 3 (C)) }\end{array}$ & 79,567 \\
\hline
\end{tabular}




\begin{tabular}{|l|l|}
\hline $\begin{array}{l}\text { Messages sent to the holding area; mostly non-positive test results (99.9\% negative) } \\
\text { (Figure 3 (D)) }\end{array}$ & 69,793 \\
\hline Messages assigned to EpiTrax for investigation (Figure 3 (F)) & 8,033 \\
\hline $\begin{array}{l}\text { Messages deleted due to irrelevant treatment, cases older than 30 days, etc. } \\
\text { (Figure 3 (E)) }\end{array}$ & 1,741 \\
\hline Total STD cases updated with clinical information (Figure 3 (G)) & $\mathbf{2 , 7 3 5}$ \\
\hline
\end{tabular}

\section{Discussion}

Despite successfully demonstrating that clinical data from EHRs can be automatically electronically transmitted directly to a public health surveillance system with little manual intervention, many challenges still exist in correctly and efficiently implementing eCR. The most common issues encountered were related to implementing correct trigger codes, defining when to trigger CCDs for multiple patient encounters, linking treatment to encounters, and understanding the structure of the EHR as it related to the public health surveillance system. Though some of what is described below is specific to this study, the lessons learned throughout the implementation of eCR in Utah can be translated to other settings and are provided as guidance for readers to note when starting to implement eCR in their own jurisdictions.

\section{Implementing Trigger Codes}

The transition of ICD codes from ICD-9 to ICD-10 presents mapping issues, as both new and existing codes need to be added to rules engines to ensure all possible triggers are included. Additionally, the use of ICD-9 or ICD-10 codes for the same disease may vary by provider; thus, an extensive list must be programmed within the EHR and the public health surveillance system, inclusive of all possible diagnostic codes that could be used. Challenges can also be presented when providers do not use a code considered useful by public health (e.g., a generic or local code that cannot be mapped to a specific disease in the public health surveillance system) or if a provider misses adding a code altogether to a patient's EHR encounter. In some limited situations, EMSA was able to leverage the ability of the rules engine to update multiple cases in the public health surveillance system simultaneously and append clinical data from trigger codes that identify a family of conditions to all cases under that family. For example, clinical data from a CCD triggered by an ICD-10 code of "Z20.2" (Contact with and (suspected) exposure to infections with a predominantly sexual mode of transmission) could be applied to both CT and GC cases under the same patient, if found. 


\section{Defining When to Trigger}

Challenges also arise when trying to determine trigger timing. For this project, these challenges were overcome by working closely with PPAU and their EHR vendor to determine at what point during a patient encounter (or a patient with multiple encounters) a provider adds a code to a patient chart and when that information should be triggered to public health. Analysis of PPAU staff workflow and usage of the EHR system led to determination of trigger timing that allows complete capture of the necessary data. This resulted in multiple ways that the trigger codes need to be checked for validity. Message generation was triggered when a relevant code was used in a new or updated encounter or when a laboratory result was entered for the person. Clinical practice also had to be modified in that all PPAU users of the EHR were trained to record ICD codes deemed usable by public health.

\section{Linking Treatment to Encounters}

The general layout of PPAU's EHR contains both a patient management system as well as a financial management system, both of which contain information specific to STD cases reported to public health. In addition, treatment information was especially problematic, as treatment administration is directly associated with a specific encounter, and situations commonly arose where multiple encounters were recorded for a single patient visit. Especially in cases where treatment was administered for prophylaxis, encounters for treatment were often distinct from encounters with confirmatory diagnoses and did not contain trigger codes that could be used to link the treatment to a specific condition. Further, because "medications prescribed" and "medications administered" were captured in multiple areas of the EHR, there was a lengthy process to correctly identify, map, and capture treatments for patients.

Issues also arose with unrelated treatments being administered in the same encounter as treatments for STDs (e.g., azithromycin and ibuprofen for CT). UDOH was able to overcome this challenge through a combination of flexible vocabulary mapping in the rules engine and by implementing restrictions within the public health surveillance system. Treatment codes reported via eCR could be mapped to treatment values that were on an approved list of treatments for specific STD conditions; those treatments that did not map to one of these treatments would then be excluded and not added to the case.

\section{Understanding the EHR Structure}

For this project, it was noted that the laboratory data transmitted from the EHR was insufficient in the CCD, as there are missing fields such as "specimen source", "collection date", and "accession number". While there was sufficient information to determine condition and test result status from laboratory data, there was not sufficient data present to reliably de-duplicate laboratory results against results that may have already been entered. Additionally, laboratory results sent from PPAU via CCD were not linked to encounters via a unique identifier specific to the encounter, and could only be loosely associated to an encounter via date. As there were often multiple encounters for the same date, this would have resulted in an excess of duplicate laboratory data for the rules 
engine to filter out. Thus, eCR in this case serves as a supplement to ELR, and was not used to directly add laboratory results to cases.

Another issue that was discovered was the use of custom templates. Since the design of an EHR system is meant to be used across many healthcare settings, capturing detailed social and sexual history at PPAU required the creation of custom templates. Those pieces of data, however, could not be exported in the CCD.

Pregnancy status presented unique challenges as the only way to link pregnancy and a positive case of disease was if the patient received a pregnancy test on the same encounter that also had a trigger code that clearly identified a CT/GC diagnosis, or with an encounter on the same date as a positive CT/GC laboratory test. Further, because pregnancy is a condition that can change over time, and investigators only need to know if a patient was pregnant at the same time as the CT and/or GC diagnosis, it was impossible to use the EHR data to set or change the pregnancy status of a case at a single point in time. Rather, a cardinal "Electronic Pregnancy Report" data element was added to relevant patients any time a relevant diagnosis code was received that would record a point-in-time pregnancy status. The list of "Electronic Pregnancy Reports" could be viewed in its entirety for all events associated with that person to allow investigators to determine pregnancy status at a single point in time relevant to the specific case in question.

\section{Limitations}

Limitations were identified during this demonstration project, several of which were previously discussed in detail. Many, although not all, of these limitations should decrease over time as eCR is more broadly implemented. The most notable limitations for the described eCR approach includes: public health being constrained by what providers send, CCD and EHR structure, dependency on correct and complete trigger code usage, and message creation timing.

Public health can only use and process what a provider can and is willing to send. The CCD was the most comprehensive and efficient standard available for this project. The EHR system was not capable of generating a message specific to public health.

The structure of the EHR and CCD limited UDOH's ability to capture all the desired information. Custom items, such as, social and sexual history, were not in a structured area of the EHR and were not included in the CCD standard and therefore could not be exported. Lab test date (and other important laboratory information) was not included in the generated CCD for this project. Several assumptions had to be made regarding the relationships of data due to the way the data were structured in both the EHR and CCD.

Exported information from an EHR is only as good as what the provider inputs. If a provider entered an incorrect code, a code that was not useful, or forgot to use a code, that information could not be exported or linked to other relevant information. The timing of when to trigger information for an event presented many issues. If a patient had multiple encounters for a single issue, defining when trigger codes must be used and messages generated could be extremely complicated. 


\section{Future Work and Opportunities}

Although challenges existed, many positive outcomes resulted from this project. In addition to realizing the initial goals of decreasing investigation time, improving the completeness, timeliness, and volume of STD data, and receiving data not provided by ELR, a better understanding and more efficient way of working was realized at UDOH and within PPAU clinics. The partnership between public health and clinical practice was strengthened, as well as a better understanding of what each group requires to work efficiently and how to work together to achieve something beneficial for all parties. This was a lasting outcome not originally included in the project design.

The eCR process created and demonstrated in this project, though limited in scope by disease, will prove to be useful for other conditions that require provider diagnosis and/or clinical symptom observation for case classification (e.g., pertussis or Zika virus). Some examples of how this eCR process can be expanded beyond communicable disease include silicosis monitoring for occupational health, opioid overdose tracking for injury prevention, and blood pressure, BMI, and blood glucose supervision for chronic disease surveillance.

This project demonstrated the feasibility of eCR from a single provider with eight clinics. eCR infrastructure was developed with the intent to enable expansion beyond small providers into larger healthcare systems. The automation capabilities demonstrated will be crucial for healthcare systems reporting more diseases and in higher volumes.

\section{Conclusion}

When this project commenced UDOH was in the distinctive position of figuring out the unknown. Though the feasibility of harnessing clinical data from EHRs and transmitting that data automatically to a public health surveillance system was demonstrated, it was also discovered that eCR is much more sophisticated and technically challenging than merely pressing a button to export information. Infrastructure needs to be created and refined (in terms of a dynamic public health surveillance database with a complex rules engine); programming needs to be analytical and on-going; clinical usage of the EHR may require provider retraining; timely communication between public health, the EHR vendor, and the clinical partner is crucial for success; and all these processes must be evaluated continuously. As a result of all these lessons learned, this demonstration project proved successful in creating a sustainable process that clinical practice can utilize to improve STD case report data in Utah. The successes achieved in this project have established a strong foundation for UDOH to receive and process clinical data via EHRs which will serve as a very important knowledge base for future work with eCR initiatives, both internally and externally. This pilot project should be seen as a model for other agencies to use when initiating eCR activities in their jurisdictions.

\section{Acknowledgements}

Penny Davies, Lesley Bailey, and Fred Pennington, Planned Parenthood Association of Utah

Kristen Kreisel and Mark Stenger, Centers for Disease Control and Prevention 
Utah Department of Health, Disease Control and Prevention Informatics Program

Utah Department of Technology Services

\section{Financial Disclosure}

Funding provided through a Cooperative Agreement with the U.S. Centers for Disease Control and Prevention, PS13-1306 STD: Surveillance Network.

\section{Competing Interests}

No Competing Interests.

\section{References}

1. Kirbiyik U, Dixon D, Grannis S. Effect of Electronic Health Record Systems Access on Communicable Disease Report Completeness. Online Journal of Public Health Informatics. ISSN 1947-2579. http://ojphi.org. 2014; 6(1).

2. Mac Kenzie W, et al. 2016. The Promise of Electronic Case Reporting. Public Health Rep. 131(6), 742-46. PubMed https://doi.org/10.1177/0033354916670871

3. Centers for Disease Control and Prevention. Sexually Transmitted Disease Surveillance 2017. Atlanta: U.S. Department of Health and Human Services; 2018.

4. Klompas M, et al. Harnessing Electronic Health Records for Public Health Surveillance. Online Journal of Public Health Informatics. ISSN 1947-2579. http://ojphi.org. 2011; 3(3).

5. Roberts E, et al. Automated Processing of Electronic Data for Disease Surveillance. Online Journal of Public Health Informatics. ISSN 1947-2579. http://ojphi.org. 2018; 10(1).

6. Birkhead G, Klompas M, Shah N. 2015. Public Health Surveillance Using Electronic Health Records: Rising Potential to Advance Public Health. Front Public Health Serv Syst Res. 4(5), 25-32. doi:10.13023/FPHSSR.0405.05.

7. Health Level Seven International. 2018. HL7 CDA R2 Implementation Guide: Consolidated CDA (C-CDA) Templates for Clinical Notes (US Realm) DSTU Release 2.1 (with errata). Available at: https://www.hl7.org/implement/standards/product_brief.cfm?product_id=408. 